\title{
Hyperspectral imaging of cuttlefish camouflage indicates good color match in the eyes of fish predators
}

Chuan-Chin Chiao $^{1,2}$, J. Kenneth Wickiser ${ }^{3}$, Justine J. Allen ${ }^{1,4}$, Brock Genter ${ }^{3}$, and Roger T. Hanlon ${ }^{1,5}$

1. Marine Biological Laboratory, Woods Hole, MA, USA

2. Department of Life Science, National Tsing Hua University, Hsinchu, Taiwan

3. Department of Chemistry and Life Science, US Military Academy, West Point, NY, USA

4. Department of Neuroscience, Brown University, Providence, RI, USA

5. Department of Ecology and Evolutionary Biology, Brown University, Providence, RI, USA

Running title: Hyperspectral imaging of animal camouflage

Keywords: reflectance, background matching, body pattern, skin coloration, Sepia

$$
\text { officinalis }
$$

Number of manuscript pages: 29

Number of tables: 0

Number of figures: 4

Number of supplementary figures: 12

The manuscript word count: 5,870

Corresponding author: Chuan-Chin Chiao, Ph.D.

Department of Life Science

National Tsing Hua University

101, Section 2, Kuang Fu Road

Hsinchu, 30013

Taiwan

Tel: 886-3-574-2464

Fax: 886-3-571-5934

E-mail: ccchiao@life.nthu.edu.tw 


\begin{abstract}
Camouflage is a widespread phenomenon throughout nature and an important anti-predator tactic in natural selection. Many visual predators have keen color perception, thus camouflage patterns should provide some degree of color matching in addition to other visual factors such as pattern, contrast, and texture. Quantifying camouflage effectiveness in the eyes of the predator is a challenge from the perspectives of both biology and optical imaging technology. Here we take advantage of Hyperspectral Imaging (HSI), which records full-spectrum light data, to simultaneously visualize color match and pattern match in the spectral and the spatial domains, respectively. Cuttlefish can dynamically camouflage themselves on any natural substrate and, despite their colorblindness, produce body patterns that appear
\end{abstract} to have high-fidelity color matches to the substrate when viewed directly by humans or with RGB images. Live camouflaged cuttlefish on natural backgrounds were imaged using HSI, and subsequent spectral analysis revealed that most reflectance spectra of individual cuttlefish and substrates were similar, rendering the color match possible. Modeling color vision of potential di- and tri-chromatic fish predators of cuttlefish corroborated the spectral match analysis and demonstrated that camouflaged cuttlefish show good color match as well as pattern match in the eyes of fish predators. These findings (i) indicate the strong potential of HSI technology to enhance studies 
of biological coloration, and (ii) provide supporting evidence that cuttlefish can

produce color-coordinated camouflage on natural substrates despite lacking color vision. 
/body

\section{Introduction}

Animal coloration plays a key role in many facets of natural and sexual selection

(1). Camouflage is a widespread phenomenon throughout nature and an important anti-predator tactic $(2,3)$. Camouflaged animals use diverse body patterns to make detection or recognition more difficult (4). However, many visual predators have keen color vision, thus camouflage should provide some degree of color matching in addition to other visual factors such as pattern, contrast, and texture.

Objective assessment of color signals in the eyes of the receivers (using point source spectrometers) has greatly advanced our understanding of visual communication and camouflage (5-12). Previous investigations of camouflage using image analysis (including spatial filtering and edge detection) provided insights into the mechanisms of visual perception of predators $(13,14)$, yet these studies suffer from the inability to assess the effectiveness of camouflage in the visual space of predators (15). Recent studies using digital photography in conjunction with color space modeling have examined body coloration in both spatial and spectral domains (16-20). In the present study, we exploit new imaging technology (HyperSpectral Imaging, HSI; Fig. 1A) to simultaneously obtain spatial and spectral data from camouflaged cuttlefish expressing Disruptive, Mottle, and Uniform body patterns on 
natural backgrounds (Fig. 1B-1D). The hyperspectral image is typically captured by scanning the two-dimensional sensor either spectrally or spatially in the third dimension to acquire the three-dimensional data cube of which the z-axis normally represents the reflectance spectrum of the corresponding point in the scene.

Camouflage is the primary defense of coleoid cephalopods (octopus, squid, and cuttlefish) and their rapidly adaptable body patterning system is among the most sophisticated in the animal kingdom (21-23). The expression of camouflaged body patterns in cuttlefish is a visually driven behavior. Previous studies have shown that certain background variables - such as brightness, contrast, edge and size of objects are essential for eliciting camouflaged body patterns (24-28). However, most cephalopods, including the cuttlefish under study, lack color perception (29-32), thus the vexing question of how they achieve colorblind camouflage still remains.

The goal of the present study was to analyze the spectral properties of cuttlefish and some natural substrates in the entire image, which allows us to directly visualize the spectral differences and to examine color matching between animal and background. More importantly, by modeling a few hypothetical visual systems of their predators, we can generate the camouflage views through the eyes of fish predators with either di- or tri-chromatic vision (Fig. 1). This approach provides a new methodology to evaluate camouflage body patterns in the luminance and 
chromatic channels of the receivers. To our knowledge, this is the first time that color and pattern matching in camouflaged animals is objectively visualized and assessed through the eyes of their potential predators using hyperspectral images. 


\section{Results}

Spectral properties of animal versus background that facilitate color match

To examine whether the reflectance spectra of certain skin components resemble

some background objects, the Spectral Angle Mapper (SAM) classification analysis of ENVI image analysis software (ITT Visual Information Solutions, Boulder, CO) was performed on the HSI-generated data cubes (see Supporting Information for details). Consistent with our previous measurements using the spectrometer (33), the reflectance spectra of some selected skin components showed typical spectral properties of cuttlefish (Figs. S1-S3). Curiously, most reflectance spectra of cuttlefish had a peak around $800 \mathrm{~nm}$ in the infrared range (IR), while natural substrates tested did not have this spectral characteristic. This color mismatch between animal and background would make cuttlefish detectable if the predators (or the sensor) had an infrared capability, but IR does not transmit far in seawater and is not thought to be used in aquatic visual systems (34). In the human visible wavelength range, however, the reflectance spectra of animal and some background areas are much alike. Using the SAM analysis, we confirmed that reflectance spectra of cuttlefish randomly resemble the background spectra. This suggests that the spectral similarity between animal and background may facilitate color match for camouflage. 
Viewing the color and pattern similarities between animal and background via di-and tri-chromatic systems of hypothetical fish predators

To examine if the spectral similarity between cuttlefish and background can impede detection by potential predators using their color vision, we modeled both diand tri-chromatic vision systems of fish predators viewing these camouflaged cuttlefish on natural substrates (Fig. 1). While the pseudo-color image gives the convenient human view of cuttlefish in shallow water (Fig. 2A), the di- and tri-chromatic composite images generated by combining the quantum catch images facilitate the visualization of cuttlefish from predators' views (Figs. 2C, 2D). By removing the luminance information (Fig. 2B) from these di- and tri-chromatic composite images (i.e., projecting the quantum catch images onto the isoluminance plane, see Methods for details), the isoluminant chromatic images (Figs. 2E, 2F) can be obtained to represent only the color information (or hue) remaining in the scene. Close examination of these chromatic images revealed that most features were washed out and the overall contrast was reduced significantly. This suggests that color information of camouflaged cuttlefish in the chromatic channels of di- and tri-chromatic fish predators is much reduced. To further characterize the chromatic discriminability $(\Delta \mathrm{S})$ of cuttlefish against background in the eyes of these predators, the color contrast images (Figs. 2G, 2H) were generated by assigning $\Delta \mathrm{S}$ between 
each pixel and averaged background in the color space of di- and tri-chromatic fish (35). These images showed that the chromatic just-noticeable-differences (JNDs) between animal and background were relatively small and distributed randomly, an indication of good color match for cuttlefish.

To simulate the effect of color change with increasing depth of water, the transmission spectra of Coastal water type $3(36)$ at $1 \mathrm{~m}$ and $10 \mathrm{~m}$ were included in the model (Fig. 1F). Similar to the images in Figure 2, color information and chromatic discriminability of camouflaged cuttlefish in the eyes of di- and tri-chromatic fish predators were also reduced significantly at $1 \mathrm{~m}$ and 10m depth (Figs. S4, S5). These results suggest that - despite the von Kries color constancy mechanism for each receptor (Eq. 2) that was implemented in the model - the chromatic information of camouflaged cuttlefish was still further reduced with water depth, which makes the visual detection by potential predators using their color vision even more difficult. A similar trend was also observed for cuttlefish in different body patterns (Figs. S6-S8 and S9-S11). Quantitative analyses further substantiated that chromatic information of camouflaged cuttlefish on all three substrate types was much less than that in the luminance channel (Fig. S12). Finally, to emphasize the importance of chromatic information in certain visual tasks, we applied the same modeling on the standard test plate for the human red-green colorblindness (Fig. 3), illustrating how the number 5 
can only be readily detected in the isoluminant tri-chromatic image, thus highlighting the importance of color information for pattern recognition. Taken together, our evidence demonstrates that the spectral similarity between cuttlefish and background can drastically reduce the effectiveness of color discrimination by their potential predators with keen color vision.

To take advantage of the HSI data, we compared the body pattern of animal and the texture pattern of background in the luminance and chromatic channels using the method we term "granularity analysis." It is apparent that the contrast (the amplitude of the curve) and the spatial scale (the shape of the curve) of animal and background were much alike in all three substrate types (Fig. 4), suggesting a good general background resemblance by these camouflaged cuttlefish. The observation that the contrast of the monochromatic images was much higher than that of the di- and tri-chromatic images also corroborates that the contrast information of cuttlefish largely resides in the luminance channel of the fish predators, not in their chromatic channels. Thus, in addition to good color match, camouflaged cuttlefish also show good pattern match in the eyes of di- and tri-chromatic fish. 


\section{Discussion}

Camouflage exploits the perceptual capabilities of predators mainly by hindering detection or recognition of the prey (15). A good deal of recent progress has been achieved in uncovering some of the mechanisms and functions of camouflage in various taxa (4). Color plays one of the key roles in camouflage but it has proved intractable to acquire images and spectra simultaneously to provide a fuller representation of the light data available in any scene at the exact time of exposure. Here we show that a new technology - HSI - provides a way to take advantage of digital imagery in such a manner that many spectra can be obtained in every pixel of the image, which then allows highly flexible post-analyses to tailor the light and pattern information to the visual system of different predators. This is a superior scientific methodology compared to using RGB digital cameras, which are designed

for human tri-chromatic vision. Yet some practical and analytical issues still require refinement before HSI can come into common use in vision research and ecology.

Hyperspectral imaging adds a new dimension to quantifying animal camouflage in the eyes of predators

To understand the adaptive features of the visual system in animal communication, it is crucial to be able to measure the visual signals (from the sender) 
as seen by the eyes of the beholder (the receiver). In the past, many studies used spectrometry to acquire chromatic information of the signals, i.e. the reflectance spectra of the color patches on the animal or the plant $(5,8,37,38)$. By mapping the spectral data onto the color space of a studied animal species based on the estimated photon catches for known photoreceptor types, insights can be gained about the strength of color signals from the viewpoint of the receiver $(7,9-11,39,40)$. However, conventional spectrometers offer only point samples, making the study of patterns (spatial relationship of sampled points) in their context difficult, if not impossible.

The potentially optimal solution for characterization of both spatial and spectral information in studying animal communication is the use of multispectral or hyperspectral imaging systems (but see a few exceptions in 16, 17-20). In the last decade, this multiband imaging system, originally designed for remote sensing, has been applied largely in studying human color vision (41-46) and animal color communication (47). In the present camouflage study, the use of HSI allows us to visualize the spectral difference and to examine color and pattern matches between animal and background through the eyes of the fish predators. This provides a new way to evaluate camouflage body patterns in spatial and spectral domains simultaneously. Despite some drawbacks of HSI (e.g., long exposure time, relatively large size and cost), future technology to improve scanning speed, camera sensitivity 
and resolution should eventually allow HSI instruments to obtain not only spatial and spectral information, but also temporal information at real time. These 4-dimensional image data $(\mathrm{x}, \mathrm{y}, \lambda, \mathrm{t})$ will ultimately change the way we study visual communication of animals in their natural habitats.

How do colorblind cuttlefish achieve color match and hide in plain sight of their visual predators?

Most animals have a fixed or slowly changing camouflage pattern, but cephalopods can rapidly adapt their skin pattern for appropriate camouflage against a staggering array of visual backgrounds (21-23). Although how cuttlefish detect visual features on the substrates and deploy appropriate body patterns to conceal themselves has been studied extensively in the laboratory (see review in 22,48 ), little is known about how cuttlefish deceive their common visual predators such as teleost fishes, diving birds, and marine mammals, which typically have di-, tri-, or even tetra-chromatic vision, in plain sight.

The color-changing abilities of cephalopods have been appreciated since Aristotle's time. Although this topic has received much attention in the past (e.g., 21, 49), no quantitative assessment has been made to examine the color match between animal and background. In the present study, we used HSI to simultaneously acquire 
spatial and spectral information of the common European cuttlefish (S. officinalis) on a small range of natural substrates with the intention of evoking the three main camouflage body pattern types (Disruptive, Mottle, and Uniform (23)). By comparing the spectral similarity across the spatial domain, and modeling the views through the di- or tri-chromatic vision systems of potential predators, we demonstrated that colorblind cuttlefish can - on certain backgrounds - achieve high-fidelity color match for camouflage. It is also noteworthy that the cuttlefish's White square stands out with higher contrast in di- and tri-chromatic images of potential fish predators (Figs. 2C, 2D); this is a tenet of disruptive coloration proposed by Cott (2) and noted in cephalopods recently (48).

It is intriguing that, despite their sophisticated color and pattern change, cuttlefish are colorblind (21, 29-32). Although our previous observations and the present study suggest that the spectral properties of some natural substrates and cuttlefish skin colors are similar (33), thus the high-fidelity color match for camouflage is achievable in the eyes of di- or tri-chromatic fish predators, how exactly they match their skin coloration to more colorfully diverse habitats (mostly shallow water environments) is still elusive.

In addition to chromatophores, cuttlefish have various structural reflectors (leucophores and iridophores) lying subjacent to chromatophores, and these 
complement color production in the skin (50). By retracting the chromatophores and thus revealing the underlying leucophores, the skin can reflect some of the ambient light, which may aid both wavelength and intensity matching (51). Furthermore, the iridophores could also alter the appearance of chromatophores by reflecting specific wavelengths (especially shorter wavelengths that the pigmented chromatophores cannot produce) through thin-film interference $(52,53)$. Given the rich repertoire of cuttlefish skin components (chromatophores, leucophores, and iridophores), it is likely that color resemblance by cuttlefish is also achieved even in the most spectrally rich environments known (e.g., kelp forests and coral reefs).

A recent discovery suggests distributed sensing of light by the skin of cuttlefish. Mäthger et al. (54) found opsin transcripts (mRNA expression) in the fin and ventral skin of S. officinalis. The single visual pigment in the cuttlefish eye is an opsin with $\lambda_{\max }=492 \mathrm{~nm}$. The opsin expressed in the fin and ventral skin is identical to that of the retina, thus color discrimination by the skin opsin is unlikely since this still renders the animal "monochromatic." Yet this finding provides a possible mechanism for distributed light sensing in the skin to adjust overall brightness match to the immediate background. Future research might find skin opsins tuned to other wavelengths, which would introduce the possibility of color sensing in the skin. 


\section{Materials and Methods}

\section{Animals and experimental setup}

Young cuttlefish (Sepia officinalis L.) ranging in size from 4 to $6 \mathrm{~cm}$ mantle length (ML), were used in this study. Each animal was placed in a tank (55 x $40 \times 15$ $\mathrm{cm})$ with flowing seawater and restricted to a cylindrical arena $(14.3 \mathrm{~cm}$ diameter and $4.9 \mathrm{~cm}$ height) where three types of natural substrates were presented on the floor (the wall was uniform gray or uniform white). These natural substrates are known to evoke the three major body patterns $(23,55)$, namely Disruptive (mixture of differently colored rocks), Mottle (gravel), and Uniform/Stipple (sand). A high-intensity lamp for faster hyperspectral image scanning, the Tungsten fluorescent solar simulator, was used to illuminate the arena evenly via a diffuser. The output of this light source (8.2 amps) was 1,500 lux, which enabled shorter exposure times for scanning. Once the animal had acclimated, the hyperspectral images were taken by the HyperScan VNIR system (Opto-Knowledge Systems, Inc., Torrance, CA) mounted $148 \mathrm{~cm}$ above the arena. There were two animals used per substrate in the experiment, and some animals were repeatedly used for different substrates. See the Supporting Information for details of Hyperspectral imaging system.

Hyperspectral imaging system and image acquisition 
The TE-cooled, low-noise CCD camera of the HyperScan VNIR system has a spatial resolution of 696 pixels in the $\mathrm{x}$ dimension, and a total of 412 spectral channels in the $\mathrm{z}$ dimension (at $1.3 \mathrm{~nm}$ interval, from $368.9 \mathrm{~nm}$ to $900.7 \mathrm{~nm}$; FWHM $2.0 \mathrm{~nm}$, $25 \mu \mathrm{m}$ slit). Using the push-broom imaging mode (scanning resolution $5 \mu \mathrm{rad}$ ), this system can acquire 580 pixels in the y dimension, with a $70 \mathrm{~mm}$ lens. The image bit depth is 12 bit, and the exposure control ranges from $10 \mu$ s to $17.9 \mathrm{~min}$. Thus, this system results in a cube of the hyperspectral image data, with $696 \times 580 \times 412$ voxels in the $x-y$ spatial dimensions and the $z$ spectral dimension, respectively (Fig. 1A).

To account for the internal noise of the imaging system, dark images were taken after each scan and subtracted out from the previously acquired radiance images. To obtain the reflectance images for modeling the chromatic information from the predator's view, the NIST Spectralon white standard was imaged soon after taking the radiance images using exactly the same camera settings. The dark-subtracted Spectralon data cube was then used to normalize the dark-subtracted radiance data cube and to derive the reflectance cube. This process allows us to obtain the reflectance spectrum (along the $\mathrm{z}$ dimension) for each pixel in the $\mathrm{x}-\mathrm{y}$ dimension. For easily visualizing these hyperspectral images, the ENVI program (a software commonly used for processing and analyzing geospatial imagery; ITT Visual Information Solutions, Boulder, CO) was used. Three represented frames $(650,550$, and $450 \mathrm{~nm}$ for R, G, and B channels, 
respectively) from the hyperspectral images were selected to form a pseudo-color image for convenient visualization. Three such pseudo-color images of cuttlefish showing Disruptive, Mottle, and Uniform body patterns on natural substrates were illustrated (Fig. 1B-1D).

\section{Modeling the predator's view}

Although the details of visual systems of cuttlefish predators are not known (Serranus cabrilla is the only fish species observed directly to prey on S. officinalis in the Mediterranean sea $(21,55))$, we chose one dichromatic fish and one trichromatic fish as their potential predators to simulate their views of these camouflaged cuttlefish.

In dichromatic fish, the $\lambda_{\max }$ of S and M cones was 450 and $545 \mathrm{~nm}$. In trichromatic fish, the $\lambda_{\max }$ of $\mathrm{S}, \mathrm{M}$, and L cones was 450,530 , and $560 \mathrm{~nm}$. It should be noted that although the choice of these $\lambda_{\max }$ of dichromatic and trichromatic cones was arbitrary, shifting the $\lambda_{\max }$ of these cones up or down 10-20 nm did not significantly affect the results.

To simulate what the fish predators see these camouflaged cuttlefish, we first computed the receptor quantum catches for each pixel of the HSI images (35). The receptor quantum catch, $q_{i}$, in photoreceptor of type $i$ is calculated as:

$q_{t}=k_{t} \int_{\lambda} I(\lambda) T(\lambda) R(\lambda) S_{t}(\lambda) d \lambda$ 
where $k_{i}$ is an arbitrary scaling factor; $\lambda$ denotes wavelength, $I(\lambda)$ is the irradiance spectrum of D65 (56); T( $\lambda$ )is the transmission spectrum of Coastal water type 3 at $1 \mathrm{~m}$ or $10 \mathrm{~m}(36) ; R(\lambda)$ is the reflectance spectrum of each pixel in the HSI image; $S_{i}(\lambda)$ is the spectral sensitivity of a receptor $i$ (57); and integration is over the range 369-700 nm (Fig. 1E-G). The $k_{i}$ describes the von Kries transformation, which is a mechanism for color constancy by independent adaptation of photoreceptors (56).

$k_{t}=1 / \int_{\lambda} I(\lambda) \mathrm{T}(\lambda) R^{b}(\lambda) S_{t}(\lambda) d \lambda$

where $R^{b}(\lambda)$ is the averaged reflectance spectrum of all pixels in the HSI image.

Following the Fechner's law, the signal of a receptor channel is proportional to the logarithm of the quantum catch, thus the coded quantum catch $Q_{i}$ in photoreceptor of type $i$ is calculated as:

$Q_{i}=\ln \left(q_{i}\right)$

Quantum catch images of four different $\lambda_{\max }$ photoreceptors (Q450, Q530, Q545, and Q560) were then used to generate composite images of cuttlefish when viewed through the eyes of di- and tri-chromatic fish predators (Fig. 1H). These were formed by assigning Blue channel as the Q450 image, and Green/Red channels as the Q545 image for dichromatic vision. Similarly, the trichromatic view was formed by assigning Blue, Green, and Red channels as the Q450, Q530, and Q560 images, respectively (16). To separate the visual information in luminance and chromatic 
channels, we assumed the luminance signal is solely from the $\mathrm{M} / \mathrm{L}$ cones in fish, thus the Q545 quantum catch image (averaged Q530 and Q560 for trichromatic vision) was used as the monochromatic image, representing the luminance information. The chromatic images were extracted from the composite images by removing luminance information and thus making the resultant images isoluminant. This was done by computing the difference between each quantum catch image from the mean of the composite image, and assigning the corresponding RGB channels accordingly (47). To estimate the chromatic discriminability $(\Delta S)$ of cuttlefish against background in the eyes of predators, the color space models of Vorobyev and Osorio (35) were implemented. Details of the equations used to derive $\Delta S$ (i.e., the just noticeable difference, JND) are given in the Supporting Information. The color contrast images were generated by assigning $\Delta \mathrm{S}$ between each pixel and averaged background in the color space of di- and tri-chromatic predators (16).

\section{Quantification of body patterns and background textures}

To characterize the body patterns of camouflaged cuttlefish, the granularity analysis $(24,58)$ was applied (see the Supporting Information for details). Briefly, the three major pattern types of cuttlefish (Disruptive, Mottle, and Uniform) differ in spatial scales (or, granularity), thus we can quantify such differences by analyzing the 
image of the animal in different spatial frequency bands. The resultant granularity spectrum of the image was then used to distinguish the body patterns. The amplitude and shape of these curves reflect high/low contrast and coarse/fine scale of the body patterns, respectively. Similarly, the granularity spectrum of background texture can be obtained using the same analysis. Processed images in the luminance and chromatic channels of di- and tri-chromatic predators (e.g., Fig. 2B, 2E, and 2F) were subjected to this granularity analysis for evaluating the pattern match between animal and background. 


\section{Acknowledgments}

We thank LTC John Ingram (Department of Chemistry and Life Science, US Military

Academy, West Point) for assistance at the beginning of the project, and the Photonics

Research Center at West Point for the support and use of the HSI equipment. Lydia

Mäthger provided helpful suggestions and we appreciate husbandry assistance from

various lab members at MBL. We gratefully acknowledge financial support from the

National Science Council of Taiwan NSC-98-2628-B-007-001-MY3 to CCC, from

the Network Science Center at West Point and the Army Research Office to JKW,

from the NDSEG Fellowship to JJA, and from ONR grant N000140610202 to RTH. 


\section{References}

1. Ruxton GD, Sherratt TN, \& Speed MP (2004) Avoiding attack: the evolutionary ecology of crypsis, warning signals, and mimicry (Oxford University Press, Oxford).

2. Cott HB (1940) Adaptive coloration in animals (Methuen \& Co., Ltd., London).

3. Thayer GH (1909) Concealing-coloration in the animal kingdom: an exposition of the laws of disguise through color and pattern: being a summary of Abbott H. Thayer's discoveries (Macmillan, New York, NY).

4. Stevens M \& Merilaita S (2009) Animal camouflage: current issues and new perspectives. Philos Trans R Soc Lond B Biol Sci 364(1516):423-427.

5. Cuthill IC, Bennett ATD, Partridge JC, \& Maier EJ (1999) Plumage reflectance and the objective assessment of avian sexual dichromatism. Am Nat 153:183-200.

6. Osorio D \& Vorobyev M (1996) Colour vision as an adaptation to frugivory in primates. Proc Biol Sci 263:593-599.

7. Stuart-Fox D \& Moussalli A (2008) Selection for social signalling drives the evolution of chameleon colour change. PLoS Biology 6(1):e25.

8. Endler JA (1990) On the measurement and classification of colour in studies of animal colour patterns. Biol J Linn Soc 41:315-352.

9. Heiling AM, Herberstein ME, \& Chittka L (2003) Pollinator attraction: crabspiders manipulate flower signals. Nature 421:334.

10. Théry M \& Casas J (2002) Predator and prey views of spider camouflage. Nature 415:133.

11. Marshall NJ \& Vorobyev M (2003) The design of color signals and color vision in fishes. Sensory Processing in Aquatic Environments, eds Collin SP \& Marshall NJ (Springer-Verlag, New York), pp 194-223.

12. Briscoe AD, et al. (2010) Positive selection of a duplicated UV-sensitive visual pigment coincides with wing pigment evolution in Heliconius butterflies. (Translated from eng) Proc Natl Acad Sci USA 107(8):3628-3633 (in eng).

13. Godfrey D, Lythgoe JN, \& Dumball DA (1987) Zebra stripes and tiger stripes: the spatial frequency distribution of the pattern compared to that of the background is significant in display and crypsis. Biol J Linn Soc 32:427-433.

14. Osorio D \& Srinivasan MV (1991) Camouflage by edge enhancement in animal coloration patterns and its implications for visual mechanisms. Proc Biol Sci 244:81-85.

15. Troscianko T, Benton CP, Lovell PG, Tolhurst DJ, \& Pizlo Z (2009) 
Camouflage and visual perception. Philos Trans R Soc Lond B Biol Sci 364(1516):449-461.

16. Chiao C-C, Wu W-Y, Chen S-H, \& Yang E-C (2009) Visualization of the spatial and spectral signals of orb-weaving spiders, Nephila pilipes, through the eyes of a honeybee. J Exp Biol 212:2269-2278.

17. Spottiswoode CN \& Stevens M (2010) Visual modeling shows that avian host parents use multiple visual cues in rejecting parasitic eggs. Proc Natl Acad Sci USA 107:8672-8676.

18. Stevens M, Stoddard MC, \& Higham JP (2009) Studying primate color: towards visual system-dependent methods. Int J Primatol 30:893-917.

19. Stoddard MC \& Stevens M (2010) Pattern mimicry of host eggs by the common cuckoo, as seen through a bird's eye. Proc Biol Sci 277:1387-1393.

20. Stevens M, Párraga CA, Cuthill IC, Partridge JC, \& Troscianko TS (2007) Using digital photography to study animal coloration. Biol J Linn Soc 90:211-237.

21. Hanlon RT \& Messenger JB (1996) Cephalopod Behaviour (Cambridge University Press, Cambridge).

22. Hanlon RT, et al. (2011) Rapid adaptive camouflage in cephalopods. Animal camouflage: mechanisms and functions, eds Stevens M \& Merilaita S (Cambridge University Press, Cambridge, U.K.).

23. Hanlon R (2007) Cephalopod dynamic camouflage. Curr Biol 17(11):R400-404.

24. Chiao C-C, Chubb C, Buresch K, Siemann L, \& Hanlon RT (2009) The scaling effects of substrate texture on camouflage patterning in cuttlefish. Vision Res 49(13):1647-1656.

25. Chiao C-C, et al. (2010) Mottle camouflage patterns in cuttlefish: quantitative characterization and visual background stimuli that evoke them. $J$ Exp Biol 213:187-199.

26. Chiao C-C, Chubb C, \& Hanlon RT (2007) Interactive effects of size, contrast, intensity and configuration of background objects in evoking disruptive camouflage in cuttlefish. Vision Res 47(16):2223-2235.

27. Zylinski S, Osorio D, \& Shohet AJ (2009) Perception of edges and visual texture in the camouflage of the common cuttlefish, Sepia officinalis. Philos Trans R Soc Lond B Biol Sci 364(1516):439-448.

28. Zylinski S, Osorio D, \& Shohet AJ (2009) Edge detection and texture classification by cuttlefish. J Vision 9(13):1-10.

29. Brown PK \& Brown PS (1958) Visual pigments of the octopus and cuttlefish. Nature 182:1288-1290. 
30. Marshall NJ \& Messenger JB (1996) Colour-blind camouflage. Nature 382:408-409.

31. Mäthger LM, Barbosa A, Miner S, \& Hanlon RT (2006) Color blindness and contrast perception in cuttlefish (Sepia officinalis) determined by a visual sensorimotor assay. Vision Res 46(11):1746-1753.

32. Bellingham J, Morris AG, \& Hunt DM (1998) The rhodopsin gene of the cuttlefish Sepia officinalis: sequence and spectral tuning. Journal of Experimental Biology 201:2299-2306.

33. Mäthger LM, Chiao C-C, Barbosa A, \& Hanlon RT (2008) Color matching on natural substrates in cuttlefish, Sepia officinalis. J Comp Physiol A Neuroethol Sens Neural Behav Physiol 194(6):577-585.

34. Cronin TW (2008) Visual ecology. The Senses: A Comprehensive Reference. Vol 1, Vision I, eds Masland RH \& Albright TD (Academic Press, San Diego), pp 211-246.

35. Vorobyev M \& Osorio D (1998) Receptor noise as a determinant of colour thresholds. Proc R Soc B 265:351-358.

36. Jerlov NG (1968) Optical oceanography (Elsevier, Amsterdam).

37. Chittka L \& Menzel R (1992) The evolutionary adaptation of flower colours and the insect pollinators' colour vision. J Comp Physiol A 171:171-181.

38. Gerald MS, Bernstein J, Hinkson R, \& Fosbury RAE (2001) Formal method for objective assessment of primate color. Am J Primatol 53:79-85.

39. Endler JA \& Mielke PWJ (2005) Comparing color patterns as birds see them. Biol J Linn Soc 86:405-431.

40. Kelber A, Vorobyev M, \& Osorio D (2003) Animal colour vision-behavioural tests and physiological concepts. Biol Rev 78:81-118.

41. Párraga CA, Troscianko T, \& Tolhurst DJ (2002) Spatiochromatic properties of natural images and human vision. Curr Biol 12:483-487.

42. Long F \& Purves D (2003) Natural scene statistics as the universal basis of color context effects. Proc Natl Acad Sci USA 100:15190-15193.

43. Nascimento SM, Ferreira FP, \& Foster DH (2002) Statistics of spatial cone-excitation ratios in natural scenes. J Opt Soc Am A 19:1484-1490.

44. Ruderman DL, Cronin TW, \& Chiao C-C (1998) Statistics of cone responses to natural images: implications for visual coding. J Opt Soc Am A 15:2036-2045.

45. Vora PL, Farrell JE, Tietz JD, \& Brainard DH (2001) Image capture: simulation of sensor responses from hyperspectral images. IEEE Transactions on Image Processing 10:307-316.

46. Wachtler T, Lee T-W, \& Sejnowski TJ (2001) Chromatic structure of natural 
scenes. J Opt Soc Am A 18:65-77.

47. Chiao C-C, Vorobyev M, Cronin TW, \& Osorio D (2000) Spectral tuning of dichromats to natural scenes. Vision Res 40:3257-3271.

48. Hanlon RT, et al. (2009) Cephalopod dynamic camouflage: bridging the continuum between background matching and disruptive coloration. Philos Trans R Soc Lond B Biol Sci 364(1516):429-437.

49. Holmes W (1940) The colour changes and colour patterns of Sepia officinalis L. Proc Zool Soc Lond A 110:2-35.

50. Mäthger LM, Denton EJ, Marshall NJ, \& Hanlon RT (2009) Mechanisms and behavioural functions of structural coloration in cephalopods. (Translated from eng) J R Soc Interface 6 Suppl 2:S149-163 (in eng).

51. Froesch D \& Messenger JB (1978) On leucophores and the chromatic unit of Octopus vulgaris. J Zool Lond 186:163-173.

52. Mäthger LM \& Hanlon RT (2007) Malleable skin coloration in cephalopods: selective reflectance, transmission and absorbance of light by chromatophores and iridophores. (Translated from eng) Cell Tissue Res 329(1):179-186 (in eng).

53. Mäthger LM \& Hanlon RT (2006) Anatomical basis for camouflaged polarized light communication in squid. Biol Lett 2:494-496.

54. Mäthger LM, Roberts SB, \& Hanlon RT (2010) Evidence for distributed light sensing in the skin of cuttlefish, Sepia officinalis. Biol Lett 6:600-603.

55. Hanlon RT \& Messenger JB (1988) Adaptive coloration in young cuttlefish (Sepia officinalis L.): The morphology and development of body patterns and their relation to behaviour. Philos Trans R Soc Lond B 320:437-487.

56. Wyszecki G \& Stiles WS (1982) Color science, concepts and methods, quantitative data and formulae, 2-nd edition (John Wiley, New York).

57. Stavenga DG, Smits RP, \& Hoenders BJ (1993) Simple exponential functions describing the absorbance bands of visual pigment spectra. Vision Res 33:1011-1017

58. Barbosa A, et al. (2008) Cuttlefish camouflage: The effects of substrate contrast and size in evoking uniform, mottle or disruptive body patterns. Vision Res 48(10):1242-1253. 


\section{Figure Legends}

Figure 1 Hyperspectral imaging and modeling of camouflaged cuttlefish through the eyes of the hypothetical di- and tri-chromatic fish predators. (A) A 3-dimensional cube of the hyperspectral image (HSI) data, in which the $\mathrm{x}$ and $\mathrm{y}$ dimensions are spatial domains, and the $\mathrm{z}$ dimension is the spectral domain. (B)-(D) Pseudo-color images of cuttlefish showing Disruptive, Mottle, and Uniform body patterns on natural substrates, respectively. (E) The irradiance spectrum $I(\lambda)$ of standard day light, D65 (56). (F) The transmission spectra $T(\lambda)$ of Coastal water type 3 at $1 \mathrm{~m}$ and $10 \mathrm{~m}$ (36). (G) The sensitivity spectra $S(\lambda)$ of given cone photoreceptors with known $\lambda_{\max }$. (H) The reflectance spectra $R(\lambda)$ from the HSI data are modeled (see Methods) for convenient visualization (human view) and for analyzing color signals through the eyes of the fish predators (animal view). Scale bar, $2 \mathrm{~cm}$.

Figure 2 Color matching of camouflaged cuttlefish when viewed by hypothetical diand tri-chromatic fish predators. (A) The pseudo-color image for simulating the human view of the cuttlefish at near-surface. This composite image was formed by using three frames $(650,550$, and $450 \mathrm{~nm})$ of the HSI data multiplied by the irradiance spectrum (Fig. 1E). (B) The monochromatic image for representing the luminance information of di- and tri-chromatic predators. (C)\&(D) The composite 
images for simulating the di- and tri-chromatic predator views of the cuttlefish, respectively. (E)\&(F) The isoluminant chromatic images for representing the color information of di- and tri-chromatic predators. $(\mathrm{G}) \&(\mathrm{H})$ The color contrast images showing just-noticeable-differences (JNDs) of color signals between animal and background when viewed by di- and tri-chromatic predators (see Methods). The scales indicate JNDs. Scale bar, $2 \mathrm{~cm}$.

Figure 3 Illustration of the importance of color information in pattern recognition. (A) The Ishihara colorblind test plate of Number 5. (B) The luminance information of the plate. This was obtained by averaging its RGB frames directly. (C)\&(D) The chromatic information of the plate under di- and tri-chromatic vision, respectively. The original RGB frames were treated as the quantum catch images of di- or tri-chromatic predators (Fig. 1H), and the isoluminant chromatic images were computed as those depicted in Fig. 2E\&2F.

Figure 4 Pattern matching of camouflaged cuttlefish versus background when viewed by hypothetical di- and tri-chromatic fish predators. (A)-(C) Average granularity spectra of cuttlefish showing Disruptive, Mottle, and Uniform body patterns, respectively, and of their corresponded substrates at near-surface. Granularity spectra 
of animals and backgrounds were obtained from images in the luminance and chromatic channels of di- and tri-chromatic predators (e.g., Fig. 2B, 2E, and 2F). The amplitude and shape of these curves reflect high/low contrast and coarse/fine scale of the body patterns, respectively. 

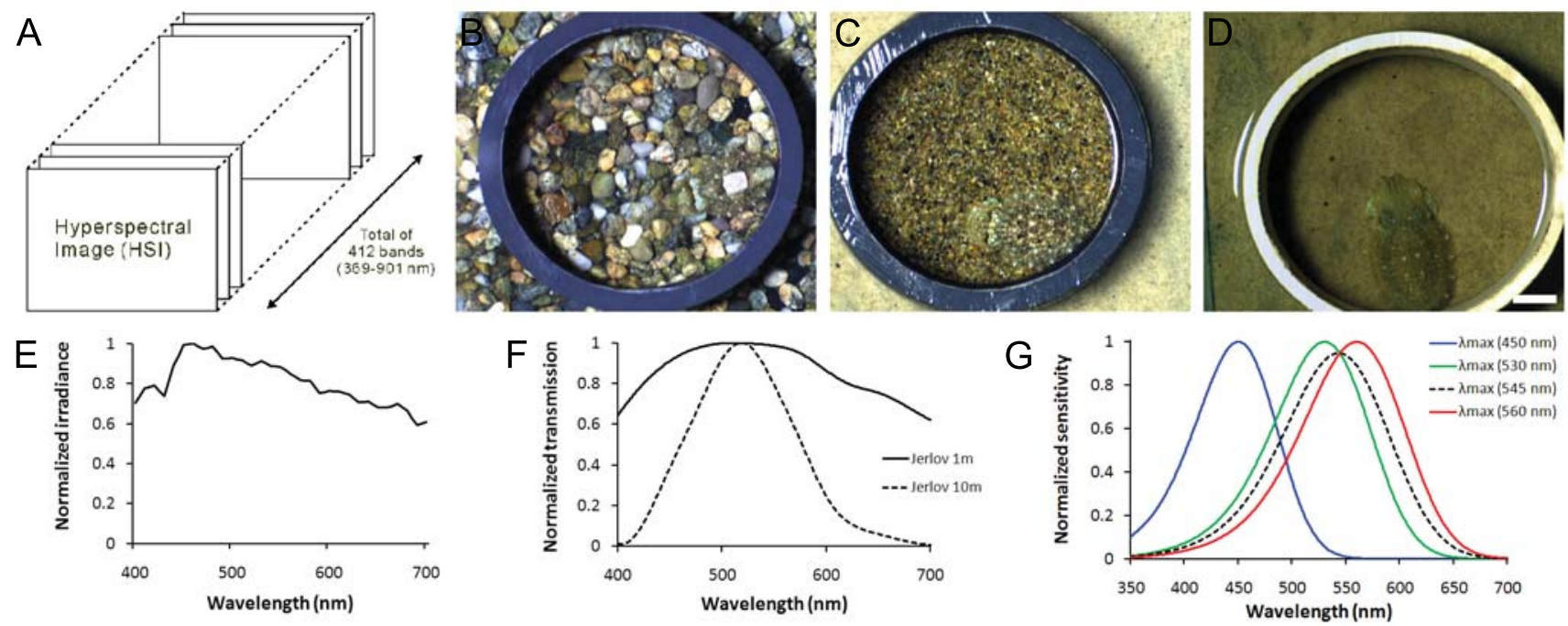

$\mathrm{H}$

Quantum catch
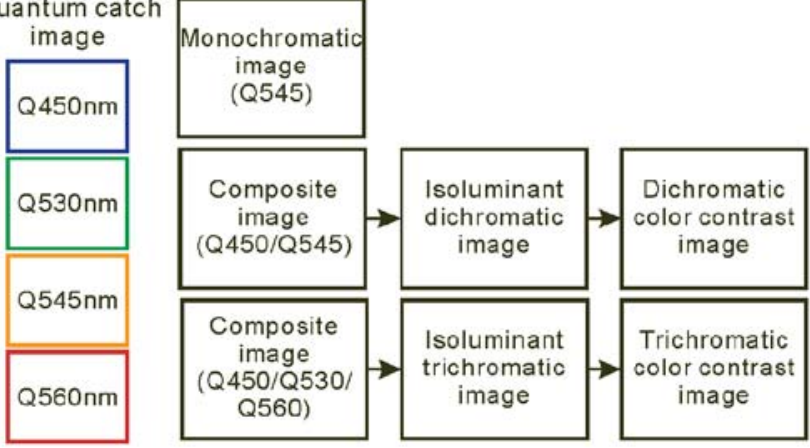

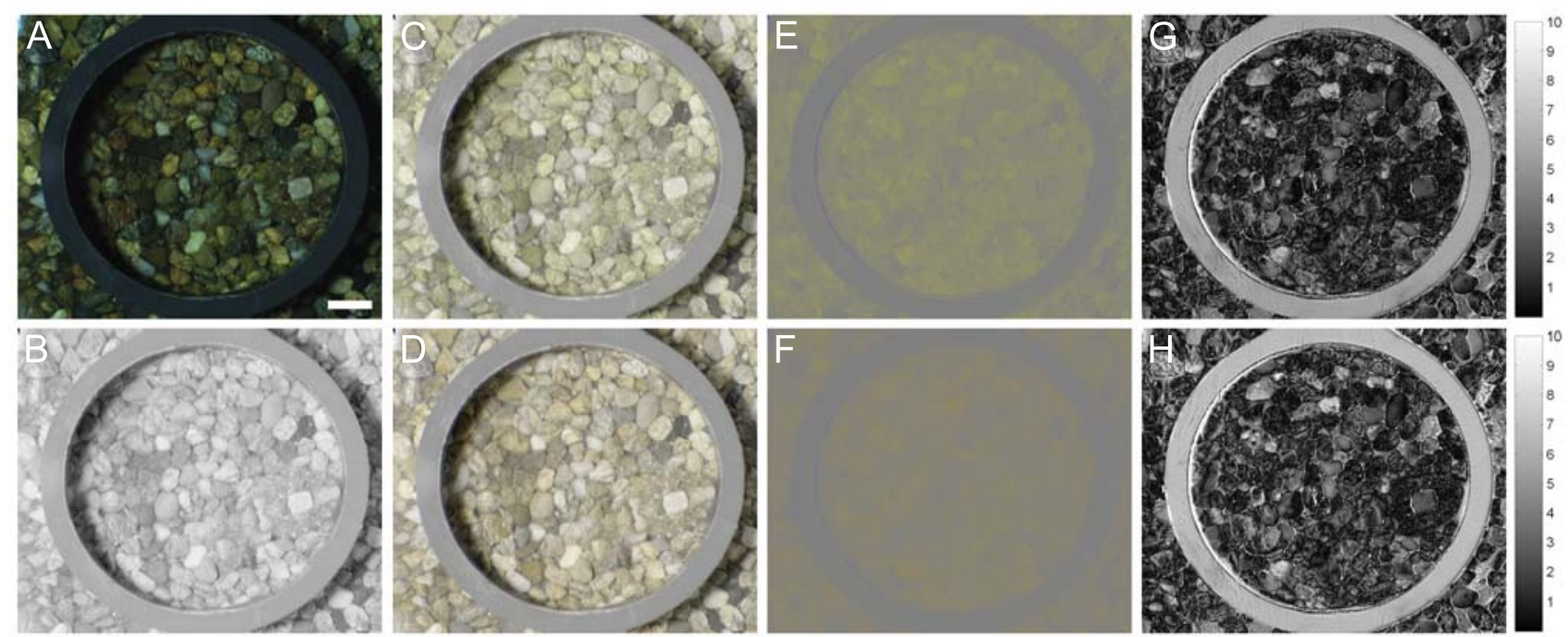


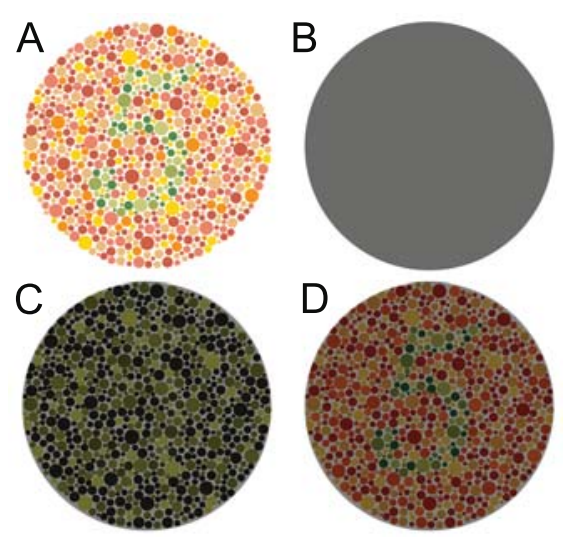



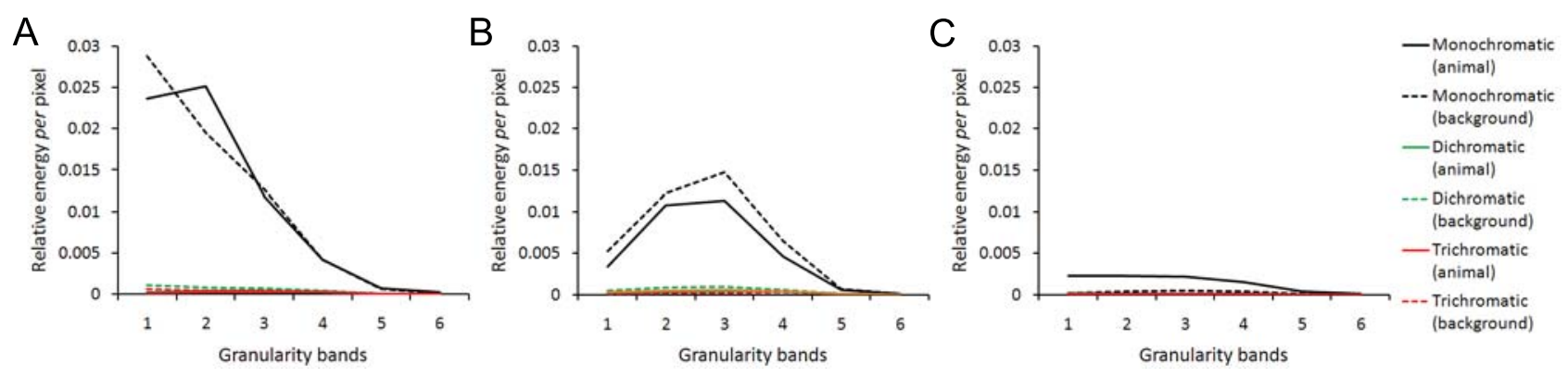